\title{
PATRONES DE ACTIVIDAD Y ABUNDANCIAS DE UNA POBLACIÓN DE LIOLAEMUS ESPINOZAI ABDALA, 2005 (IGUANIA: LIOLAEMIDAE) EN CAMPO EL ARENAL, CATAMARCA, ARGENTINA
}

\author{
María Paula CABRERA ${ }^{\mathbf{1}}$ y Gustavo José SCROCCHI ${ }^{1,2}$ \\ ${ }^{1}$ Instituto de Herpetología-Fundación Miguel Lillo-, Miguel Lillo 251, 4000. San Miguel de Tucumán, \\ Tucumán, Argentina, <mpaucab@yahoo.com> \\ ${ }^{2}$ CONICET, Buenos Aires, Argentina.
}

Cabrera, M. P. y Scrocchi, G. J. 2014. Patrones de actividad y abundancias de una población de Liolaemus espinozai Abdala, 2005 (Iguania: Liolaemidae) en Campo El Arenal, Catamarca, Argentina. Acta Zoológica Mexicana (n.s.), 30(3): 459-470.

RESUMEN. Se investigaron los patrones de actividad y abundancias relativas de Liolaemus espinozai, especie endémica de Campo El Arenal (Catamarca, Argentina), entre el año 2007 y 2010. Se contabilizó un total de 1694 avistajes (865 adultos, 620 juveniles y 209 infantiles). Los machos adultos presentan actividad entre octubre y mayo, las hembras entre noviembre y mayo; los juveniles están activos durante todo el año y los infantiles se incorporan a la población a fines de enero o principios de febrero por lo que ésta es la época con mayor abundancia total. Los adultos fueron más abundantes en primavera y verano, en tanto que los juveniles en otoño. Los machos adultos son más abundantes que las hembras durante la primavera (época reproductiva), mientras que éstas lo son en el verano (post-reproductiva). Durante los meses de mayores temperaturas (noviembre a enero) se observa un patrón de actividad bimodal y en los meses restantes la actividad es unimodal. Los patrones de actividad encontrados en adultos machos y hembras, juveniles e infantiles se relacionan de manera diferente con la temperatura, el fotoperíodo y la precipitación.

Palabras clave: Liolaemus espinozai, actividad, abundancia, Catamarca.

Cabrera, M. P. \& Scrocchi, G. J. 2014. Activity patterns and abundance of a population of Liolaemus espinozai Abdala, 2005 (Iguania: Liolaemidae) in Campo el Arenal, Catamarca, Argentina. Acta Zoológica Mexicana (n.s.), 30(3): 459-470.

ABSTRACT. Activity patterns and relative abundances were investigated of Liolaemus espinozai, an endemic species from Campo El Arenal (Catamarca, Argentina), between 2007 and 2010. There were a total of 1694 sightings (865 adults, 620 juveniles and 209 hatchlings). Male adults are active from October, followed by females one month later until May; juveniles are active throughout the year, and hatchlings are added to the population in late January or early February, so this is the time with the greatest total abundance. Adults were more abundant in spring and summer, and juveniles in autumn. Adult males are more abundant than females in spring (breeding season), while these are in the summer

Recibido: 04/06/2013; aceptado: 10/04/2014 
(post-reproductive season). The species exhibits a bimodal pattern of activity in the months of higher temperatures, and unimodal in the rest of the months. The different activity patterns found in adult males and females, juveniles and hatchlings are related in a different way with temperature, photoperiod and rainfall.

Key words: Liolaemus espinozai, activity, abundance, Catamarca.

\section{INTRODUCCIÓN}

En hábitats climáticamente variables las lagartijas presentan diferencias en los patrones de actividad (Schoener 1970; Barbault 1977), con lo que pueden hacer frente a la variabilidad temporal y espacial de la disponibilidad de recursos térmicos (Bauwens et al. 1996; Labra et al. 2001). Numerosas características de la población y de las historias de vida de las lagartijas pueden variar entre especies de clima tropical y templado y entre especies de clima húmedo y seco o árido (Rocha 1998). Las variaciones estacionales de una serie de factores extrínsecos tales como las precipitaciones, la disponibilidad de recursos alimenticios, y las temperaturas ambientales producen variaciones en los aspectos ecológicos, como el uso del espacio, la temperatura del cuerpo, y los períodos de actividad diaria, especialmente en ecosistemas templados (Pianka 1986; Angert et al. 2000; Zaluar \& Rocha 2000; Ribeiro et al. 2009). Así, las poblaciones que viven en zonas áridas o semiáridas con estacionalidad marcada por la precipitación, tienden a mostrar variación estacional en tamaño y densidad de la población, crecimiento, mortalidad, distribución de clases de edad y la frecuencia de oviposición. Además de deberse a factores físicos (precipitaciones y temperaturas entre otros), las variaciones en la actividad pueden deberse a factores ecológicos, como presión de depredación, comportamiento social y gastos energéticos relacionados con el modo de forrajeo (Etheridge \& Wit 1993; Rocha 1998; Van Sluys 2000).

Existe documentación sobre la actividad de una especie dentro de la estructura de la comunidad (Videla \& Puig 1994; Fitzgerald et al. 1999; Vega 1999; Acosta et al. 2000, 2001; Dias y Rocha 2004; Faria \& Araujo 2004; Winne \& Keck 2004; Pérez \& Balta 2007; Frutos 2009). El estudio y relevamiento de información sobre patrones de actividad y abundancias relativas en especies de Liolaemus es relativamente reciente (Ortiz 1981; Fitzgerald et al. 1999; Martori et al. 1998, 2002; Villavicencio et al. 2002; Halloy \& Robles 2003; Robles \& Halloy 2008; Belver et al. 2010; Martori \& Aun 2010). Estos análisis pueden aportar información valiosa para la determinación de los factores biológicos y ambientales que influyen en los patrones de actividad de los individuos; por lo tanto, resultan importantes tanto en un marco teórico comparativo como en un marco práctico, ya que permiten detectar potenciales problemas a través de cambios en los números observados (e.g. Rocha \& Bergallo 1992), para eventualmente formular estrategias de manejo y conservación de estas especies (Martori et al. 2002).

Liolaemus espinozai es una especie de tamaño mediano (longitud hocico-cloaca -LHC, aproximadamente 57.9 mm; 49.5 - 62.8 mm). Es insectívora, alimentándose 
principalmente de hormigas (Abdala 2005). De acuerdo a su descripción original sería vivípara, pero Cabrera \& Scrocchi (datos no publicados) indican que en realidad es ovípara. Su distribución se encuentra restringida a localidades del SE de los Nevados del Aconquija, a lo largo de Campo El Arenal y dentro de éste, en las localidades de El Ingenio y Morro El Arenal, provincia de Catamarca, Argentina, entre 2200 y 2800 $\mathrm{m}$ de altitud; en ambientes de grandes arenales y dunas, ecotono entre las provincias fitogeográficas del monte y prepuna (Cabrera 1976) en la que abundan los cardones y jarillas (Abdala 2005).

El objetivo de este estudio es describir y analizar la variación mensual y estacional en las abundancias de los distintos sexos y grupos etarios, comparar el patrón de actividad entre infantiles, juveniles y adultos y evaluar la relación entre variables climáticas (temperatura, precipitación y fotoperíodo) y el patrón de actividad de cada grupo etario.

\section{MATERIAL Y MÉTODOS}

El sitio de estudio está ubicado en Campo El Arenal (2707’04.9” S, 66¹3’04.4” W, 2462 msnm), departamento Andalgalá, provincia de Catamarca. Para evaluar la actividad temporal diaria y su variación estacional se utilizaron dos metodologías diferentes, transectas durante el primer año y grilla durante el segundo; los dos métodos arrojan los mismos resultados en cuanto a actividad y proporción de sexos y edad por lo que los resultados de ambos años son tomados en conjunto para su análisis.

Se utilizaron transectas de 500 metros de largo por 10 metros de ancho, seleccionadas al azar y todos los días en lugares distintos a fin de evitar el acostumbramiento de los individuos a nuestra presencia. Se recorrió mensualmente durante tres días sucesivos, desde diciembre de 2007 hasta enero de 2009.

La grilla se ubicó en una parcela de 70 x 70 m en la que se construyó una cuadrícula ortogonal, subdividida en cuadrantes de siete metros de lado, se recorrió en febrero, abril, mayo, noviembre y diciembre de 2009 y enero de 2010, durante tres o cuatro días seguidos por mes, realizando tres monitoreos diarios (mañana, siesta y tarde). Dentro de la cuadrícula y antes de iniciar el estudio, los ejemplares adultos fueron capturados, pesados, medidos y marcados mediante mostacillas de colores (suturadas a la base de la cola con una hebra de monofilamento de acero quirúrgico), y se les asignó una letra (M para machos y $\mathrm{H}$ para hembras) y un número (por orden de captura), según la metodología propuesta por Fisher \& Muth (1989). Posteriormente fueron liberados en el mismo sitio de captura. Tanto en las transectas como en la grilla se usó una planilla diaria en la que se registró hora de captura, sexo y grupo etario, definidos a priori como infantiles (hasta $35 \mathrm{~mm}$ o sin diferenciación de coloración entre machos y hembras), juveniles (desde 36 a 44 mm y con dimorfismo de color evidente) y adultos (mayores a $45 \mathrm{~mm}$ ) basados en datos no publicados de los autores. 
Para evaluar la relación entre las frecuencias encontradas con factores físicos se realizó una correlación de Pearson entre las frecuencias mensuales de cada grupo etario y la temperatura (temperatura media mensual), precipitación (precipitación media mensual) y fotoperíodo. Los datos de temperatura del aire y precipitación fueron obtenidos de la estación meteorológica del aeropuerto de La Alumbrera (274'36.99" S, 66³5’37.20” O); los datos del fotoperíodo fueron obtenidos del sitio web www. tutiempo.net

Para probar si la frecuencia de lagartijas observada fue similar a la esperada dentro de cada grupo analizado, se llevó a cabo test de Chi-cuadrado; cuando fue necesario los datos fueron transformados siguiendo el siguiente criterio: los valores 0 se transformaron a $1 \mathrm{y}$ luego todos los valores fueron multiplicados por la constante 10 (Villavicencio et al. 2002). Se consideró como frecuencia esperada, para todos los casos, el promedio de frecuencias observadas de individuos entre categorías. La diferencia de abundancias de adultos entre época reproductiva y pos-reproductiva se analizó con test no paramétrico de Mann Whitney.

\section{RESULTADOS}

Se obtuvo un total de 1694 avistajes durante los dos años de muestreo, de los cuales 865 fueron adultos (427 machos y 438 hembras), 620 juveniles (328 machos y 292 hembras) y 209 infantiles. El número de ejemplares marcados dentro de la grilla fue de 17 machos adultos y 23 hembras adultas. El mes que más abundancia de lagartijas presentó fue febrero, debido a la incorporación de la mayoría de los infantiles a la población.

Las hembras adultas fueron más abundantes que los machos en febrero $\left(\chi^{2}=6.05\right.$, g.l. $=1 ; \mathrm{P}=0.0139)$ y marzo $\left(\chi^{2}=5.23\right.$, g.l. $\left.=1 ; \mathrm{P}=0.0223\right)$, y los machos fueron más abundantes que las hembras en noviembre $\left(\chi^{2}=8.67\right.$, g.l. $\left.=1 ; \mathrm{P}=0.0032\right)$. A su vez, los machos fueron más abundantes en la época reproductiva (octubre, noviembre y diciembre) y las hembras más abundantes en la post-reproductiva (enero, febrero y marzo) (Mann-Whitney: $\mathrm{U}=62121 ; \mathrm{P}<0,0000 ; \mathrm{n}=395$ machos y $\mathrm{n}=387$ hembras) (Fig. 1).

Los adultos fueron más abundantes que los juveniles en los meses de enero, febrero, marzo y diciembre, mientras que los juveniles lo fueron desde mayo a octubre, y en noviembre se encontraron abundancias similares (Cuadro 1). Cuando se comparan las abundancias de los adultos y los juveniles por estación, se encuentran diferencias significativas en verano, otoño y primavera, con adultos más abundantes en primavera $\left(\chi^{2}=8.45 ;\right.$ g.l. $\left.=1 ; \mathrm{P}=0.0037\right)$ y verano $\left(\chi^{2}=14.47\right.$; g.l. $\left.=1 ; \mathrm{P}=0.0001\right)$ y juveniles más abundantes en otoño $\left(\chi^{2}=10.67\right.$; g.l. $\left.=1 ; \mathrm{P}=0.0011\right)$.

$\mathrm{Al}$ discriminar por sexo las abundancias mensuales de los juveniles, sólo se encuentran diferencias significativas en octubre, con machos más abundantes $\left(\chi^{2}=9.28\right.$; g.l. = 1; $\mathrm{P}=0.0023$ ); lo que coincide con las diferencias significativas en la primavera 


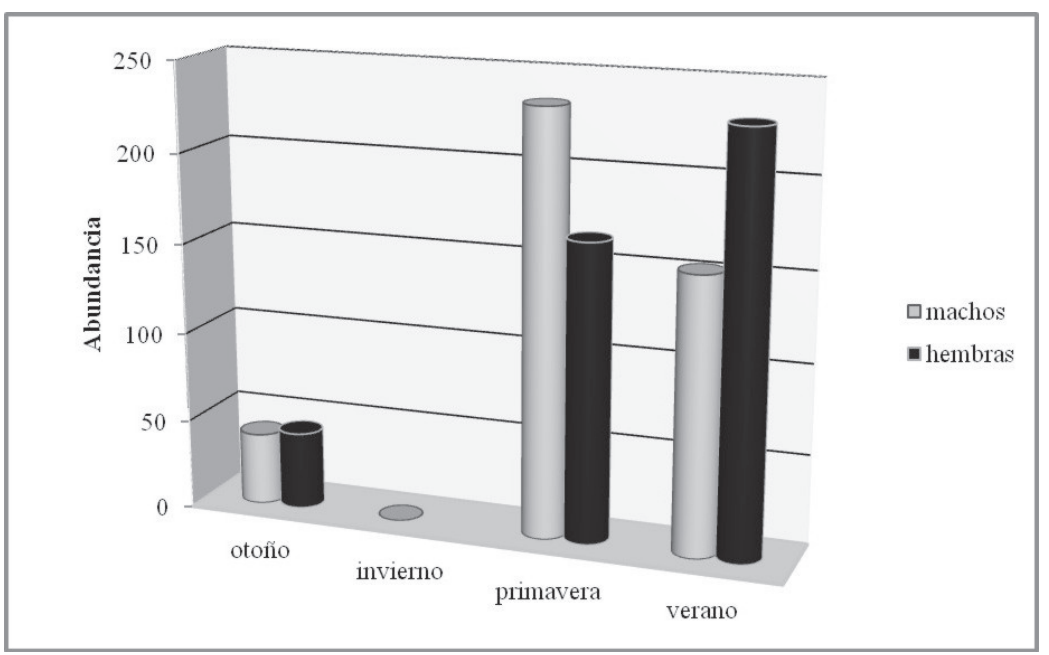

Figura 1. Abundancia por estación de machos y hembras adultos de Liolaemus espinozai.

$\left(\chi^{2}=5.24 ;\right.$ g.l. $\left.=1 ; \mathrm{P}=0.021\right)$ con machos más abundantes, correspondiéndose también con la época reproductiva.

La temperatura mínima del aire a partir de la cual los animales empiezan su actividad varía en las distintas estaciones del año, pero en general los infantes aparecen a partir de una temperatura del aire de $14^{\circ} \mathrm{C}$, los juveniles a partir de $15^{\circ} \mathrm{C}$, mientras que la temperatura mínima a la que los adultos empiezan su actividad es a los $17^{\circ} \mathrm{C}$. La máxima temperatura del aire en la que se encontraron infantiles aun activos fue a los $29^{\circ} \mathrm{C}$ mientras que los juveniles y adultos permanecieron activos hasta una temperatura máxima de $32^{\circ} \mathrm{C}$. La mayoría de las lagartijas activas (67\%) se encuentran

Cuadro 1. Comparación de abundancias relativas de adultos contra juveniles de Liolaemus espinozai por mes con el estadístico Chi Cuadrado de Pearson. En itálica valores significativos de P, g.l.= grados de libertad.

\begin{tabular}{lccc}
\hline Mes & $\chi^{2}$ & g.l. & $\mathrm{P}$ \\
\hline Enero & 4.17 & 1 & 0.0413 \\
Febrero & 12.57 & 1 & 0.0004 \\
Marzo & 13.76 & 1 & 0.0002 \\
Mayo & 5.12 & 1 & 0.0236 \\
Octubre & 20.28 & 1 & $<0.0001$ \\
Noviembre & 0.00 & 1 & $>0.9999$ \\
Diciembre & 17.12 & 1 & $<0.0001$ \\
\hline
\end{tabular}


entre 20 a $26^{\circ} \mathrm{C}$, y dentro de este intervalo, la mayor frecuencia de individuos activos (42\%) fue registrada entre los 21 y $22^{\circ} \mathrm{C}$.

En el verano, el primer individuo avistado fue una hembra juvenil a las 8:30 hs, a una temperatura de $17.62^{\circ} \mathrm{C}$ y el último fue un macho adulto a las $19: 45$ hs a $24^{\circ} \mathrm{C}$. En el otoño el primer individuo avistado fue un infantil a las $9: 48 \mathrm{hs}$ a $18^{\circ} \mathrm{C}$ y el último fue un macho adulto a las $18: 45 \mathrm{hs}$ a $18^{\circ} \mathrm{C}$. En el invierno el primer individuo avistado fue un macho juvenil a las $11: 45$ hs a $9^{\circ} \mathrm{C}$ y el último fue un infantil a las 18:30 hs a $7^{\circ} \mathrm{C}$. En primavera el primer individuo fue un macho juvenil a las 8:20 hs a una temperatura de $17^{\circ} \mathrm{C}$ y el último fue también un macho juvenil a las 19:15 hs a $26^{\circ} \mathrm{C}$ (Fig. 3).

Se encontró una relación positiva y significativa entre la frecuencia mensual de machos adultos y el fotoperíodo y temperatura pero no con la precipitación. En hembras adultas todas las correlaciones fueron positivas y significativas, en juveniles solo se encontró relación entre la frecuencia de estos con la temperatura, mientras que en los infantiles no hubo relación entre ninguna de estas tres variables climáticas y la frecuencia observada (Fig. 2).

En primavera y verano la actividad es bimodal para todos los grupos etarios, mientras que en otoño e invierno la actividad es unimodal (Fig. 3).

\section{DISCUSIÓN}

Los individuos de Liolaemus espinozai comienzan su actividad anual a principios de octubre, con la emergencia de la hibernación de los machos adultos, seguida por las hembras en noviembre; entre fines de enero y principios de febrero se incorporan los infantiles; los juveniles se encuentran activos durante todo el año. Los adultos están activos hasta principios de mayo, y a partir de este mes sólo se encuentran infantiles y juveniles durante la época fría, es decir que se observan patrones de actividad e inactividad que dependen del sexo y de la edad.

La actividad es un compromiso entre la necesidad de obtener pareja y alimento, y la de escapar de la predación y la competencia (Huey 1982; Pianka 1986; Heatwole \& Taylor 1987). En zonas templadas, los períodos con temperaturas óptimas para la actividad son limitados y, por lo tanto, el factor térmico ejerce una notable influencia sobre los ciclos de actividad (Grant \& Dunham 1988). De acuerdo a esto, se pueden obtener las siguientes conclusiones y efectuar algunas consideraciones sobre la actividad de Liolaemus espinozai.

En machos la actividad es muy alta en la época reproductiva, mientras que en la post-reproductiva son más activas las hembras. La mayor actividad de los machos en la época reproductiva podría estar relacionada con dos factores: por un lado con la necesidad de estos de definir su territorio antes de la emergencia de las hembras (Stamps 1983); y por otro, la emergencia temprana de los machos puede ser importante ya que el comportamiento de asoleo ayudaría a la maduración del esper- 

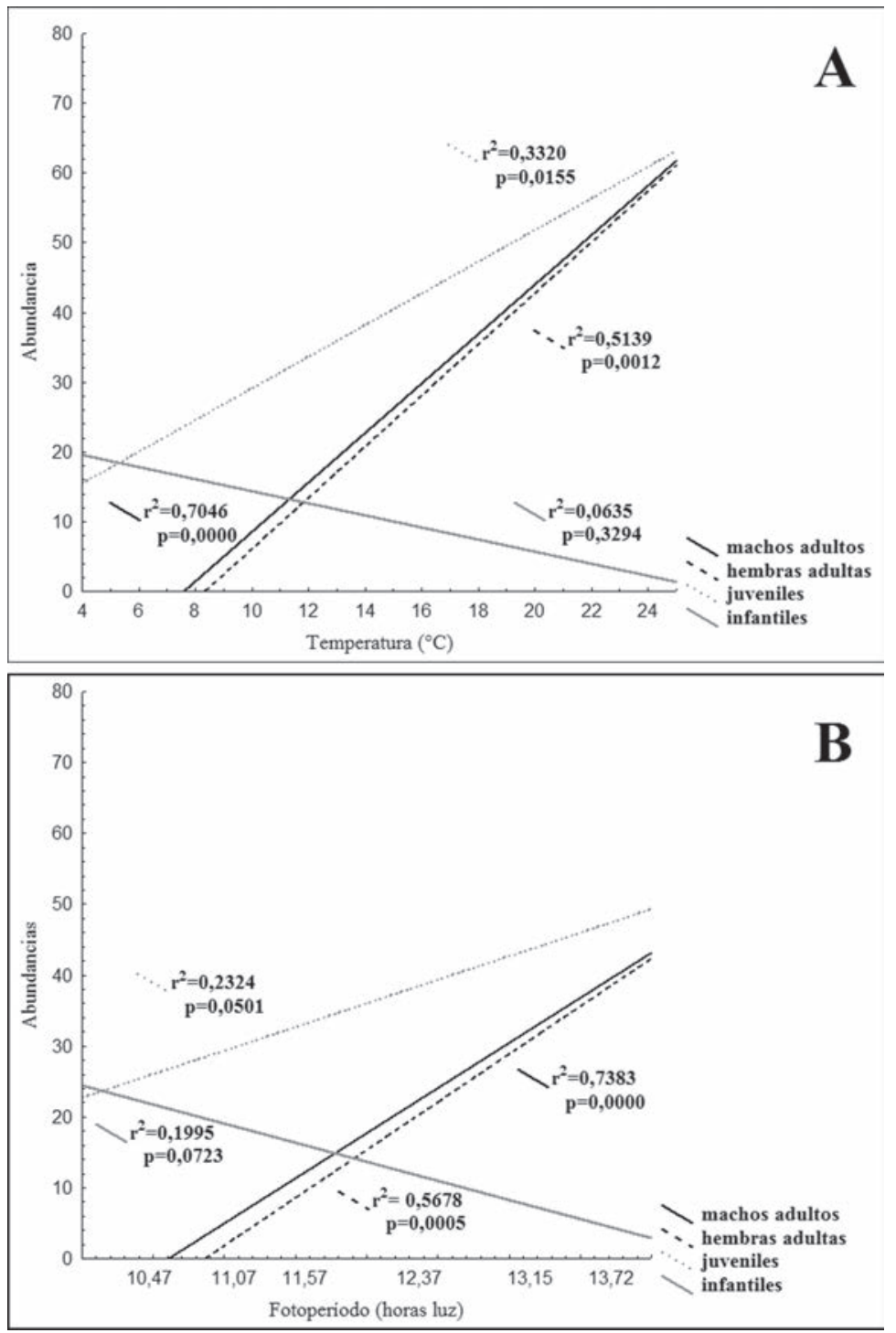

Figura 2. Correlación entre abundancias mensuales de cada grupo etario y variables climáticas, temperatura (A), fotoperíodo (B) y precipitación (C).

ma, permitiendo que estos estén reproductivamente listos cuando salgan las hembras (Saint-Girons 1985; Olsson \& Madsen 1996). Esta conclusión está reforzada por la estrecha relación entre la frecuencia de los machos adultos con la temperatura y el fotoperíodo. 

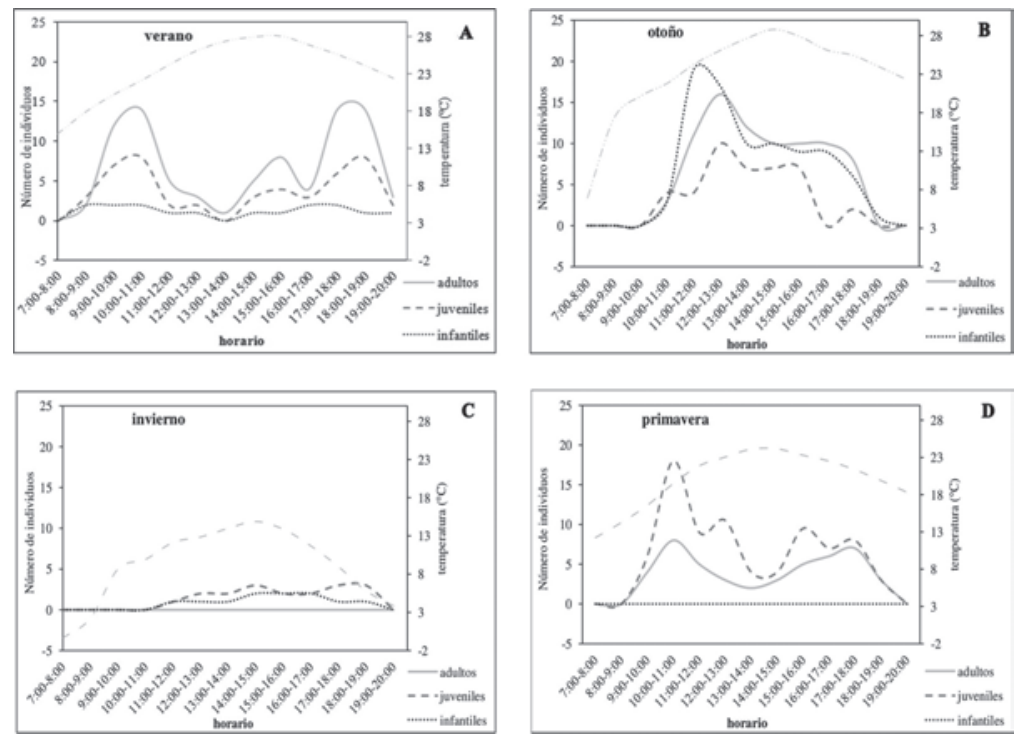

Figura 3. Patrones de actividad estacional de los distintos grupos etarios de Liolaemus espinozai, verano (A), otoño (B), invierno (C) y primavera (D).

También podría tratarse de una combinación de necesidades etológicas y fisiológicas relacionadas entre sí, tendientes a la optimización del tiempo de la reproducción en los machos. Ramírez Pinilla (1992) observó en una población de Liolaemus quilmes, que los machos eran reproductivos de septiembre a noviembre mientras que las hembras lo eran en octubre. En esa misma época del año, Halloy (1996) observó machos de la misma especie realizar despliegues asociados a encuentros agonísticos entre machos y a establecimiento de territorios (Halloy \& Robles 2002). En estos meses donde necesitan patrullar su área y cortejar a las hembras, los machos se expondrían fuertemente a los predadores, es decir que prima la reproducción frente al riesgo de predación, por lo que el descenso de la actividad en la época post-reproductiva sería una forma de reducir la presión de depredación, como se observó en otras especies (Rose 1981; Etheridge \& Wit 1993).

La mayor abundancia de las hembras durante la época post-reproductiva estaría relacionada con un aumento de la actividad de forrajeo luego de la oviposición para reponer el peso perdido, antes de entrar en letargo.

La actividad reproductiva de Liolaemus espinozai (datos de los autores, no publicados) estaría asociada con las variables físicas estudiadas: el período reproductivo se produce cuando las temperaturas son más favorables para la incubación de los huevos y el reclutamiento cuando la temperatura y la precipitación brindan un ambiente más adecuado, con mayor oferta térmica y trófica para los neonatos. 
Los patrones de actividad en adultos de Liolaemus espinozai coinciden con los de L. quilmes (Halloy \& Robles, 2003), sugiriendo que estos patrones podrían ser comunes a otras especies de lagartijas diurnas en áreas equivalentes. Sin embargo, $L$. koslowskyi no presenta este mismo patrón de actividad (Belver et al. 2010; Martori \& Aun 2010) a pesar de la estrecha relación filogenética entre estas tres especies y de la semejanza entre sus hábitats. Tampoco L. pseudoanomalus (Villavicencio et al. 2003) presenta patrones similares, por lo que existirían otros factores que influyen sobre los patrones de actividad de estas lagartijas, como la interacción con otras especies, por ejemplo.

El patrón observado en juveniles, donde los machos están más activos que las hembras a principios de la primavera, podría deberse a la necesidad de búsqueda y establecimiento de territorios, además del alimento, por lo que las temperaturas favorables beneficiarían la dispersión de los machos jóvenes.

Por otro lado, las hembras juveniles se mantendrían inactivas para evitar temperaturas ambientales extremas, disminuir el riesgo de predación y conservar energía asignándola al crecimiento. Para Rose (1981), los niveles de inactividad son parte de la estrategia adaptativa de las lagartijas, siendo tan importantes como los niveles de actividad para la supervivencia y éxito reproductivo. De acuerdo a nuestros resultados, el único factor climático que influye en la actividad de los juveniles es la temperatura; al no estar listos para la reproducción, ni la precipitación ni el fotoperíodo ejercerían influencia alguna.

No existe relación entre la frecuencia mensual de infantiles y la temperatura, la precipitación o el fotoperíodo, ya que necesariamente, al nacer deben buscar el alimento para crecer y sobrevivir al invierno. La única necesidad física de los infantiles sería alcanzar la temperatura mínima para la actividad.

Los juveniles e infantiles son los primeros en estar activos durante las horas de la mañana, mientras que los adultos están activos hasta las últimas horas del día. Según Carothers et al. (1998), esto se debería a la inercia térmica ya que los individuos pequeños pueden ganar calor más rápidamente, mientras que los de mayor tamaño lo pierden más lentamente. Una explicación alternativa y/o complementaria para este patrón de actividad diferente entre adultos y juveniles e infantiles sería la de minimizar la competencia intraespecífica, tal como fue observado por varios autores (entre ellos Simon \& Middendorf 1976; Creusere \& Whitford 1982).

El patrón de actividad registrado corresponde al patrón general descripto para las distintas especies de lagartijas en zonas templadas, con una tendencia a una actividad unimodal en los meses templados y bimodal en los meses de mayor amplitud térmica (Dias et al. 2004; Faria \& Araujo 2004; Winne \& Keck 2004; Vieira Peloso et al. 2008). Estos resultados concuerdan con el patrón observado en varias especies de Liolaemus como por ejemplo: L. darwinii (Videla \& Puig 1994; Ontivero et al. datos no publicados), L. multimaculatus (Vega 1999), L. bibronii, L. rothi, 
Cabrera \& Scrocchi: Actividad y abundancia en Liolaemus espinozai, Catamarca, Argentina

L. petrophilus y L. cf. chehuachekenk (Frutos 2009), y L. koslowskyi (Belver et al. 2010).

AGRADECIMIENTOS. A la Fundación Miguel Lillo por la ayuda financiera y a la Dirección de Fauna de Catamarca por los permisos para el trabajo de campo. A López Vázquez, G. (Bajo La Alumbrera - Copper) por brindar los datos meteorológicos. Al Dr. Félix Cruz por los comentarios y sugerencias realizados a la primera versión de este manuscrito. A Nicolás Riera, Mariana Iruzubieta y Gabriela Salva por su ayuda en el trabajo de campo.

\section{LITERATURA CITADA}

Abdala, C. S. 2005. Una nueva especie del género Liolaemus perteneciente al complejo darwinii (Iguania: Liolaemidae) de la provincia de Catamarca, Argentina. Revista Española de Herpetología, 19: 5-17.

Barbault, R. 1977. Étude comparative des cyles journaliers d'activité des lézards Cophosaurus texanus, Cnemidophorus scalaris, Cnemidophorus tigris dans le Désert de Mapimi (Mexique). Bulletin de la Société Zoologique de France, 102: 159-168.

Bauwens, D., Hertz, P. E. \& Castilla, A. M. 1996. Thermoregulation in a Lacertid lizard: the relative contributions of distinct behavioral mechanism. Ecology, 77: 1818-1830.

Begon, M., Harper, J. L. \& Townsend, C. R. 1986. Ecology. Individuals, Populations, and Communities. Sinauer Associates, Inc. Sunderland.

Belver, L.C., Kozykariski, M. \& Avila, L. J. 2010. Diferencias sexuales y etarias en la actividad diaria y estacional de una población de Liolaemus koslowskyi (Liolaemini). Cuadernos de Herpetología, 24: 71-79.

Cabrera, A. L. 1976. Regiones fitogeográficas argentinas. Enciclopedia Argentina de Agricultura y Jardineria. Ed. Acme, Buenos Aires.

Carothers, J. H., Marquet, P. A. \& Yaksic, F. 1998. Thermal ecology of a lizard assemblage along an Andean altitudinal gradient in Chile. Revista Chilena de Historia Natural, 71: 39-50.

Creusere, F.M. \& Whitford, W. G. 1982. Temporal and spatial resource partitioning in a Chihuahuan desert lizard community, pp. 12-127. In: N.J. Scott, Jr. (Ed.). Herpetological Communities, Wildlife Research Report. No 13. Fish and Wildlife Service, Washington.

Caruccio, R., Vieira, R. C., Verrastro, L. \& Machado, D. M. 2011. Thermal biology, activity, and population parameters of Cnemidophorus vacariensis (Squamata, Teiidae), a lizard endemic to southern Brazil. Iheringia, Série Zoologia, 101: 283-295.

Dias, E. J. R. \& Rocha, C. F. D. 2004. Thermal ecology, activity patterns, and microhabitat use by two sympatric whiptail lizards (Cnemidophorus abaetensis and C. ocellifer) from Northeastern Brazil. Journal of Herpetology, 38: 586-588.

Etheridge, K. \& Wit, L. C. 1993. Factors affecting activity in Cnemidophorus, pp. 151-162. In: J. Wright y L. Vitt (Eds.). Biology of Whiptail Lizards (genus Cnemidophorus). Oklahoma Museum of Natural History. Norman, Oklahoma.

Faria, R. G. \& Araujo, A. F. B. 2004. Syntopy of two Tropidurus lizards species (Squamata: Tropiduridae) in a rocky cerrado habitat in central Brazil. Brazilian Journal of Biology, 64: 775-786.

Fisher, M. \& Muth, A. 1989. A technique for permanently marking lizards. Herpetological Review, 20: 45-46.

Fitzgerald, L. A., Cruz, F. B. \& Perotti, G. 1999. Phenology of a lizard assemblage in the dry Chaco of Argentina. Journal of Herpetology, 33: 526-535. 
Frutos, N. 2009. Dominio vital, movimiento y ritmo de actividad en una comunidad de saurios patagónicos del clado Liolaemini: un análisis evolutivo. Tesis Doctoral. Facultad de Ciencias Exactas, Físicas y Naturales. Universidad Nacional de Córdoba. Argentina.

Grant, B. \& Dunham, A. E. 1988. Thermally imposed time constraints on the activity of the desert lizard Sceloporus merriami. Ecology, 69: 167-176.

Halloy, M. 1996. Behavioral patterns in Liolaemus quilmes (Tropiduridae), a South American lizard. Bulletin of Maryland Herpetological Society, 32: 43-57.

Halloy, M. \& Robles, C. 2002. Spatial distribution in a neotropical lizard, Liolaemus quilmes (Liolaemidae): site fidelity and overlapping among males and females. Bulletin of Maryland Herpetological Society, 38: 118-129.

Halloy, M. \& Robles, C. 2003 Patrones de actividad y abundancias relativas en un lagarto del noroeste argentino, Liolaemus quilmes (Iguania: Liolaemidae). Cuadernos de Herpetología, 17: 65-71.

Heatwole, H. 1970. Thermal ecology of the desert dragon, Amphibolurus inermis. Ecological Monographs, 40: 425-457.

Heatwole, H. F. \& Taylor, J. 1987. Ecology of reptiles. Surrey Beatty \& Sons, Sydney.

Huey, R. B. 1982. Temperature, physiology, and the ecology of reptiles, pp. 25-91. In: C. Gans and F.H. Pough (Eds.). Biology of the Reptilia. Vol. 12. Phisiology (C). Academic Press, New York.

Huey, R. B., Pianka, E. R. \& Hoffman, J. A. 1977. Seasonal variation in thermoregulatory behavior and body temperature of diurnal snakes and its thermal consequences. Ecology, 70: 689-692.

Labra, A., Soto-Gamboa, M. \& Bozinovic, F. 2001. Behavioral and physiological thermoregulation of Atacama desert-dwelling Liolaemus lizards. Ecoscience, 8: 413-420.

Martins, E. P., Bissell, A. N. \& Morgan, K. K. 1998. Population differences in a lizard communicative display: evidence for rapid change in structure and function. Animal Behaviour, 56: 1113-1119.

Martori, R., Cardinale, L. \& Vignolo, P. 1998. Growth in a population of Liolaemus wiegmannii (Squamata: Tropiduridae) in Central Argentina. Amphibia-Reptilia, 19: 293-301.

Martori, R., Juárez, R. \& Aún, L. 2002. La taxocenosis de lagartos de Achiras, Córdoba, Argentina: parámetros biológicos y estado de conservación. Revista Española de Herpetología, 16: 73-91.

Martori, R. \& Aún, L. 2010. Reproducción y variación de grupos de tamaño en una población de Liolaemus koslowskyi (Squamata: Liolaemini). Cuadernos de Herpetología, 24: 39-55.

Nicholson, K. L., Torrence, S. M., Ghioca, D. M., Bhattacharjee, J., Andrei, A. E., Owen, J., Radke, N. J. A. \& Perry, G. 2005. The influence of temperature and humidity on activity patterns of the lizards Anolis stratulus and Ameiva exsul in the British Virgins Islands. Caribbean Journal of Science, 41: 870-873.

Olsson, M. \& Madsen, T. 1996. Cost of mating with infertile males selects for late emergence in female sand lizards (Lacerta agilis). Copeia, 1996: 462-464.

Ortiz, J. C. 1981. Révision taxonomique et biologie des Liolaemus du groupe nigromaculatus (Squamata, Iguanidae). Thèse de Doctorat d'Etats Sciencies Naturelles, Université Paris VII.

Pérez Z., J. \& Balta K., K. 2007. Ecología de la comunidad de saurios diurnos de la Reserva Nacional de Paracas, Ica, Perú. Revista Peruana de Biología, número especial 13: 169-176.

Pianka, E. R. 1969. Sympatry of desert lizard (Ctenotus) in Western Australia. Ecology, 50: 1012-1030.

Pianka, E. R. 1986. Ecology and natural history of desert lizards: analyses of ecological niche and community structure. Princeton University Press, Princeton.

Pianka, E. R. \& Vitt, L. J. 2003. Lizards: Windows to the Evolution of Diversity. University of California Press, Berkeley.

Pimm, S. L \& Redfearn, A. 1988. The variability of population densities. Nature, 334: 613-614.

Ramírez Pinilla, M. P. 1992. Ciclos reproductivos y de cuerpos grasos en dos poblaciones de Liolaemus darwinii (Reptilia: Sauria: Tropiduridae). Acta Zoológica Lilloana, 42: 41-49. 
Robles, C. \& Halloy, M. 2008. Seven-year relative abundance in two syntopic neotropical lizards, Liolaemus quilmes and L. ramirezae (Liolaemidae), from northwestern argentina. Cuadernos de Herpetología, 22: 73-79.

Rocha, C. F. 1998. Population dynamics of the endemic tropidurid lizard Liolaemus lutzae in a tropical seasonal restinga habitat. Ciência e Cultura, Journal of the Brazilian Association for the Advancement of Science, 50: 446-451.

Rocha, C. F. D. \& Bergallo, H. G. 1992. Population decrease: The case of Liolaemus lutzae, an endemic lizard of Southeastern Brazil. Ciência e Cultura, Journal of the Brazilian Association for the Advancement of Science, 44: 52-54.

Rose, B. 1981. Factor affecting activity in Sceloporus virgatus. Ecology, 62: 706-716.

Saint-Girons, H. 1985. Comparative data on Lepidosaurian reproduction and some time tables, pp. 35-58. In: C. Gans and F. Billett (Eds.). Biology of the Reptilia, Vol. 15 B. John Wiley and Sons, New York.

Schoener, T. W. 1970. Nonsynchronous spatial overlap of lizards in patchy habitats. Ecology, 51: 408418.

Schoener, T. W. 1977. Competition and the niche, pp. 35-136. In: C. Gans and D.W. Tinkle (Eds.). Biology of Reptilia. Vol. 7. Academic Press, New York.

Simon, C. A. \& Middendorf, G. A. 1976. Resource partitioning by an iguanid lizard: temporal and microhabitat aspects. Ecology, 57: 1317-1320.

Stamps, J. 1983. Sexual selection, sexual dimorphism, and territoriality, pp. 169-204. In: R.B Huey, E.R. Pianka and T.W. Schoener (Eds.), Lizard Ecology. Harvard University Press, Cambridge.

Van Sluys, M. 2000. Population dynamics of the saxicolous lizard Tropidurus itambere (Tropiduridae) in a seasonal habitat of Southeastern Brazil. Herpetologica, 56: 55-62.

Vega, L. E. 1999. Ecología de saurios arenícolas de la dunas costeras bonaerenses. Tesis doctoral. Facultad de Ciencias Exactas y Naturales. Universidad Nacional de Mar del Plata. Argentina.

Videla, F. \& Puig, S. 1994. Estructura de una comunidad de lagartos del monte. Patrones de uso espacial y temporal. Multequina, 3: 99-112.

Villavicencio, J., Acosta, J. C., Cánovas, M. G. \& Marinero, J. A. 2002. Patrones de actividad temporal diaria y estacional de Liolaemus pseudoanomalus (Squamata: Tropiduridae) en el centro-oeste de la Argentina. Multequina 11: 51-60.

Wiens, J. A. 1997. Metapopulation dynamics and landscape ecology, pp. 43-62. In: I.A. Hanski, and M.E. Gilpin (Eds.). Academic Press, San Diego.

Winne, C. T. \& Keck, M. B. 2004. Daily activity patterns of whiptail lizards (Squamata: Teiidae): a proximate response to environmental conditions or an endogenous rhythm? Functional Ecology, 18: 314-321. 


\section{FE DE ERRATAS}

En la figura 2, faltó incluir la gráfica correspondiente sobre la Correlación entre abundancias mensuales de cada grupo etario y la precipitación.

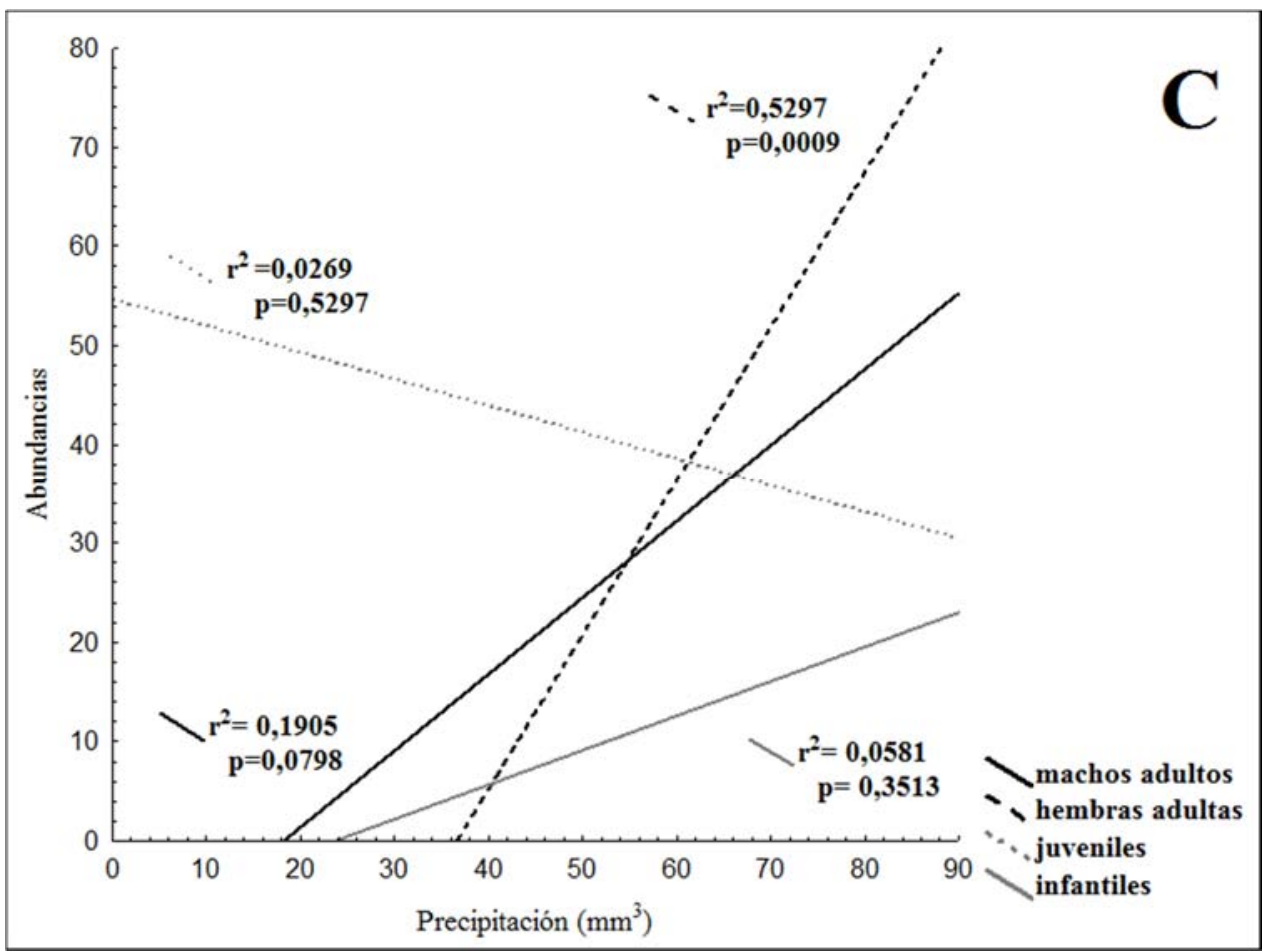

